\title{
Significance of Low Maternal Serum B-hCG Levels in the Assessment of the Risk of Atypical Chromosomal Abnormalities
}

\author{
Robin Wijngaard $^{\mathrm{a}} \quad$ Elena Casals $^{\mathrm{a}} \quad$ Imma Mercadé $^{\mathrm{a}} \quad$ Javier Laguna ${ }^{\mathrm{a}}$ \\ Irene Madrigal ${ }^{a, b}, c \quad$ Celia Badenas ${ }^{a, b}, c \quad$ Antoni Borrell ${ }^{d}$ \\ Laia Rodriguez-Revenga ${ }^{a, b, c}$
}

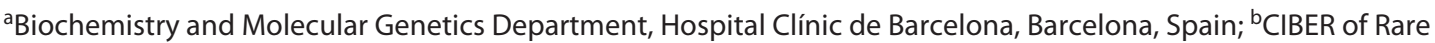
Diseases (CIBERER), Instituto de Salud Carlos III, Madrid, Spain; 'Institut d'Investigacions Biomèdiques August Pi i Sunyer (IDIBAPS), Barcelona, Spain; ${ }^{B}$ CNatal Department of Maternal-Fetal Medicine, Institute Gynecology, Obstetrics and Neonatology, Hospital Clínic de Barcelona, Barcelona, Spain

\section{Mini Summary}

- The residual risk of a clinically significant copy number variants is 3 times higher when low free betahuman chorionic gonadotropin ( $\beta$-hCG) levels or 2 times higher when fetal nuchal translucency (NT) $\geq 3.5 \mathrm{~mm}$ are detected in pregnancies at high combined first-trimester screening (cFTS) risk $(\geq 1 / 250)$.

- Free $\beta$-hCG levels and fetal NT should be considered in conjunction with the cFTS risk score to decide whether to proceed with cell-free DNA testing or invasive testing.

\section{Keywords}

Chromosomal abnormalities · Combined first-trimester screening · Noninvasive prenatal diagnosis · Free $\beta$-human chorionic gonadotrophin

\begin{abstract}
Introduction: The introduction of prenatal cell-free DNA as a screening test has surpassed traditional combined first-trimester screening (cFTS) in the detection of common trisomies. However, its current limitation in detecting only common trisomies is affecting the diagnostic yield for other clinically significant chromosomal abnormalities. Methods: In
\end{abstract}

efforts to optimize the detection of fetuses with genetic abnormalities, we have analyzed the relationship between the cFTS risk score and biomarkers with atypical chromosomal abnormalities. Furthermore, we have evaluated the impact of prenatal cell-free DNA screening on the detection of chromosomal abnormalities in our population. For these purposes, we performed a retrospective study of 877 singleton pregnancies who underwent chromosomal microarray analysis (CMA) between 2013 and 2020 and for whom cFTS data were available. Results: The results demonstrated that low levels of free beta-human chorionic gonadotropin ( $\beta-h C G)$ ( $\leq 0.37$ multiples of the median) and increased fetal nuchal translucency (NT) ( $\geq 3.5 \mathrm{~mm}$ ) were statistically associated
C 2021 The Author(s).

Published by S. Karger AG, Basel

This is an Open Access article licensed under the Creative Commons Attribution-NonCommercial-4.0 International License (CC BY-NC) (http://www.karger.com/Services/OpenAccessLicense), applicable to the online version of the article only. Usage and distribution for commercial purposes requires written permission.
Correspondence to:

Laia Rodriguez-Revenga, lbodi@ clinic.cat 
with the presence of atypical chromosomal abnormalities. In fact, the risk of pathogenic CMA results increased from 6 to $10 \%$ when fetal NT was increased and from 6 to $20 \%$ when a low serum $\beta$-hCG level was detected in the high-risk cFTS group. Moreover, our results showed that altered serum levels of $\beta$-hCG can have a substantial impact on the early detection of clinically relevant copy number variants. Discussion/Conclusion: Traditional cFTS can potentially identify a substantial proportion of atypical chromosomal aberrations, and women with increased NT or low maternal serum $\beta$-hCG levels are at increased risk of having pathogenic CMA results. Our results may help clinicians and women decide whether invasive testing or prenatal cell-free DNA screening testing is more appropriate for each situation.

(c) 2021 The Author(s).

Published by S. Karger AG, Basel

\section{Introduction}

Combined first-trimester screening (cFTS), which combines maternal age, fetal nuchal translucency (NT) and maternal serum-free beta-human chorionic gonadotropin ( $\beta$-hCG), and pregnancy-associated plasma protein-A (PAPP-A) at 11-13 weeks of gestation, continues to be the standard screening strategy for Down syndrome (trisomy 21), Edwards syndrome (trisomy 18), and Patau syndrome (trisomy 13) in many countries [1]. The cFTS has been proven to detect $90 \%$ of trisomies 21 and $95 \%$ of trisomies 18 and 13, with a false positive rate of 5\% [2-5]. All 3 trisomies are associated with increased maternal age, increased fetal NT, and decreased maternal serum PAPP-A, but serum free $\beta$-hCG is increased in trisomy 21 while it is decreased in trisomies 18 and 13 [3-5].

Noninvasive prenatal testing for the detection of fetal aneuploidy by analysis of cell-free DNA (cfDNA) in maternal blood has been introduced into national prenatal screening programs and has changed clinical practice. The efficacy of prenatal cfDNA testing for the common autosomal trisomies exceeds that of conventional screening [6]. It has been shown that cfDNA for fetal aneuploidy testing has a detection rate for trisomy 21 of over $99 \%$, with a false positive rate as low as $0.1 \%[6,7]$. Thus, prenatal cfDNA testing appears to represent a substantial improvement over traditional multiple marker screening.

The best way to apply prenatal cfDNA analysis in the general prenatal screening for aneuploidy remains controversial, and currently, worldwide national screening programs have adopted different strategies [8]. The 2 main approaches are as a second-line screening test, offered to women at high risk (secondary screening) or at intermediate risk (contingent strategy) through routine screening tests, or as a first-line screening test (primary screening), offered to the general obstetric population, i.e., all pregnant women regardless of the results from other screening tests. Since prenatal cfDNA analysis remains a screening test, women with a positive result still require confirmatory invasive testing.

As shown in other studies, trisomy 21 cases comprise slightly more than $50 \%$ of the aneuploidies present in the population $[9,10]$ and the relative proportions of atypical chromosome aberrations have been reported to be between 12 and $27 \%$ of all chromosomal aberrations detected [11-14]. The high specificity of prenatal cfDNA screening reduces the overall need for women to undergo invasive testing, avoiding the procedure-related risks, including pregnancy loss and discomfort for the woman, but which in turn may result in a lower detection rate of atypical chromosomal aberrations that are currently undetectable by cfDNA screening [15-17].

In contrast to prenatal cfDNA screening, cFTS can identify risk for a broad array of abnormalities [11-13, 18]. Further studies are needed to address the incremental value of cFTS for the detection of atypical aneuploidies, including copy number variants (CNVs), structural anomalies, and other adverse perinatal outcomes. The aims of this study were to investigate the association between cFTS risk scores and the presence of abnormal findings on chromosomal microarray analysis (CMA), to explore whether some of its markers may be useful in identifying women at high risk of CNVs and to assess the impact of prenatal cfDNA screening on detection of $\mathrm{CNVs}$ in our population.

\section{Materials and Methods}

This retrospective study included data from all singleton pregnancies followed up at our institution between 2013 and 2020 that underwent cFTS for common aneuploidies and CMA testing, on either amniotic fluid or chorionic villi. This involves a heterogenous group of pregnant women undergoing invasive testing for different reasons, including cFTS risk, ultrasound (US) abnormalities, familiar or personal history, or personal choice. No cases of fetal aneuploidy potentially detectable by prenatal cfDNA screening (trisomies 21, 18, and 13, and sex chromosome aneuploidies) were included. In addition, all cases of benign, likely benign, and variants of unknown significance according to the American College of Medical Genetics and Genomics [19] and susceptibility CNVs detected by CMA testing were excluded. Both cFTS testing and invasive testing were carried out in full compliance with the protocol for prenatal screening of congenital anomalies in Catalonia, which is a free of charge prenatal screening program available to all pregnant women in Catalonia. 


\section{Combined First-Trimester Screening}

Prenatal screening was offered to women attending our institution, or associated primary care facilities, in the first 14 weeks of pregnancy. This program included maternal serum biomarker analysis (PAPP-A and $\beta$-hCG) and US for NT measurement.

According to the guidelines of the prenatal screening program, blood samples for biomarker analysis were drawn between 8 and $13+6$ weeks of pregnancy. Samples were immediately centrifuged and frozen until the next workday's analysis. PAPP-A and $\beta$-hCG were determined on a Delfia Xpress analyzer (Perkin-Elmer, Waltham, MA, USA), a biochemical fluoroimmunoassay analyzer. Absolute PAPP-A and $\beta$-hCG concentrations were transformed into gestational age-specific multiples of the median (MoM). Additionally, US measurement of NT was performed between $11+2$ and $13+6$ weeks of gestation.

Risk scores for Down syndrome, Edwards syndrome, and $\mathrm{Pa}$ tau syndrome were calculated using LifeCycle v3.0 ${ }^{\circledR}$ software, including the aforementioned biomarkers, fetal NT, and maternal age. The cFTS risk scores were stratified into the following groups: very high risk $(>1 / 10)$, high risk $(1 / 10-1 / 250)$, intermediate risk $(1 / 251-1 / 1,100)$, and low risk $(<1 / 1,100)$.

\section{Invasive Testing}

Invasive testing, on either amniotic fluid or chorionic villi, was conducted in a sequential manner. At first, quantitative fluorescence polymerase chain reaction (QF-PCR) was used to detect common trisomies (trisomies 21, 18, and 13) and sex chromosome aneuploidies, with karyotype confirmation when any of these alterations were present. If common trisomies were ruled out, CMA testing was performed by array comparative genomic hybridization CNV detection. An olignonucleotid analyzing chromosomal regions associated with recognized clinical syndromes from $\mathrm{qGe}-$ nomics (qChipCM $8 \times 60 \mathrm{~K}$, qGenomics, Barcelona, Catalonia, Spain) was used. This microarray has a genome-wide resolution of $350-500 \mathrm{~kb}$, a resolution of $100-125 \mathrm{~kb}$ in subtelomeric and pericentromeric zones, and a resolution of $30 \mathrm{~kb}$ in constitutional regions associated with pathology. This microarray applies to the University of California Santa Cruz Genome Browser hg19 as the reference assembly. Samples were hybridized against a sexmatched reference DNA sample. Images were processed and analyzed by Cytogenomics software (Agilent Technologies) and qGenviewer software (qGenomics).

\section{Ethical Approval}

The study was approved by the Institutional Ethical Committee on January 24, 2020 (HCB/2019/1029).

\section{Statistical Analyses}

Data on cFTS biomarkers and risk scores, along with indications for invasive testing and CMA results of the included women, were gathered from the laboratory data system and electronic health records. The study population was divided into 2 groups according to the CMA results (normal or pathogenic CMA result). The frequency of abnormal NT $(\geq 3.5 \mathrm{~mm})$ and abnormal low or high maternal serum analytes was calculated for both groups. Biomarker cutoff points were based on the 5th (p5) and 95th (p95) percentile values obtained from 3,970 singleton pregnancies undergoing cFTS at our center throughout 2018 and 2019. These percentiles were: $0.36 \mathrm{MoM}$ and 2.46 MoM for PAPP-A and 0.37 $\mathrm{MoM}$ and 2.51 MoM for $\beta$-hCG. Frequencies were compared us-

Low Serum $\beta$-hCG Levels and the Risk of CNVs ing Fisher's exact test. Odds ratio (OR) with 95\% confidence interval (CI) was calculated to assess the correlation between groups and outcome. A $p$ value of less than 0.05 was considered statistically significant. For convenience, when risk score is mentioned across this article, it refers specifically to the Down syndrome risk score.

\section{Results}

A total of 943 singleton pregnancies in whom cFTS and CMA was performed at our institution during the study period were examined. Of this, 66 were excluded due to benign, likely benign, variants of unknown significance, susceptibility variants, or sex chromosome aneuploidies, leaving 877 for analysis. Abnormal CMA results accounted for 45 (5\%) of the 877 included cases.

Figure 1 shows the reasons for invasive diagnostic tests of the entire study population. A total of 233 women (27\%) underwent invasive testing as consequence of an increased cFTS risk score, 57 of them (25\%) had simultaneous US abnormalities. Among women at low risk $(<1 / 250)$, the most frequent indication was an US abnormality in 496 cases (77\%), including malformations, fetal growth restriction, polyhydramnios, and hydrops fetalis. In our cohort of abnormal CMA results, 32 had a low cFTS risk score $(71 \%)$ whereas 13 had a high cFTS risk score $(29 \%)$. Finally, 2\% (1/45) of the clinically relevant aberrations were detected in pregnancies with no indication for an invasive procedure (personal choice).

The number of cases stratified according cFTS risk score, abnormal serum analytes, and increased fetal NT is summarized in Table 1. Characterization of significant CNVs according to cFTS risk score groups is displayed in supporting information online supplementary Table S1 (see www.karger.com/doi/10.1159/000521345 for all online suppl. material).

The frequencies of abnormal serum analytes $(\leq \mathrm{p} 5$ and $\geq$ p95) and increased fetal NT ( $\geq 3.5 \mathrm{~mm})$ were compared between the groups of women with and without atypical chromosomal aberrations. A statistically significant 2.5fold increased risk (OR 2.62, 95\% CI, 1.17-5.89, $p$ value $=$ 0.02 ) and an almost significant 2 -fold increased risk (OR $2.18,95 \%$ CI, $0.98-4.86$, $p$ value $=0.06$ ) of encountering clinically relevant $\mathrm{CNV}$ were identified in women with low $\beta$-hCG levels and increased fetal NT, respectively (Table 2). In contrast, neither increased $\beta$-hCG levels nor altered PAPP-A levels were associated with an increased risk of pathogenic CNVs in CMA testing (Table 2).

The prevalence and residual risks of an atypical chromosomal aberration were calculated for women with a 


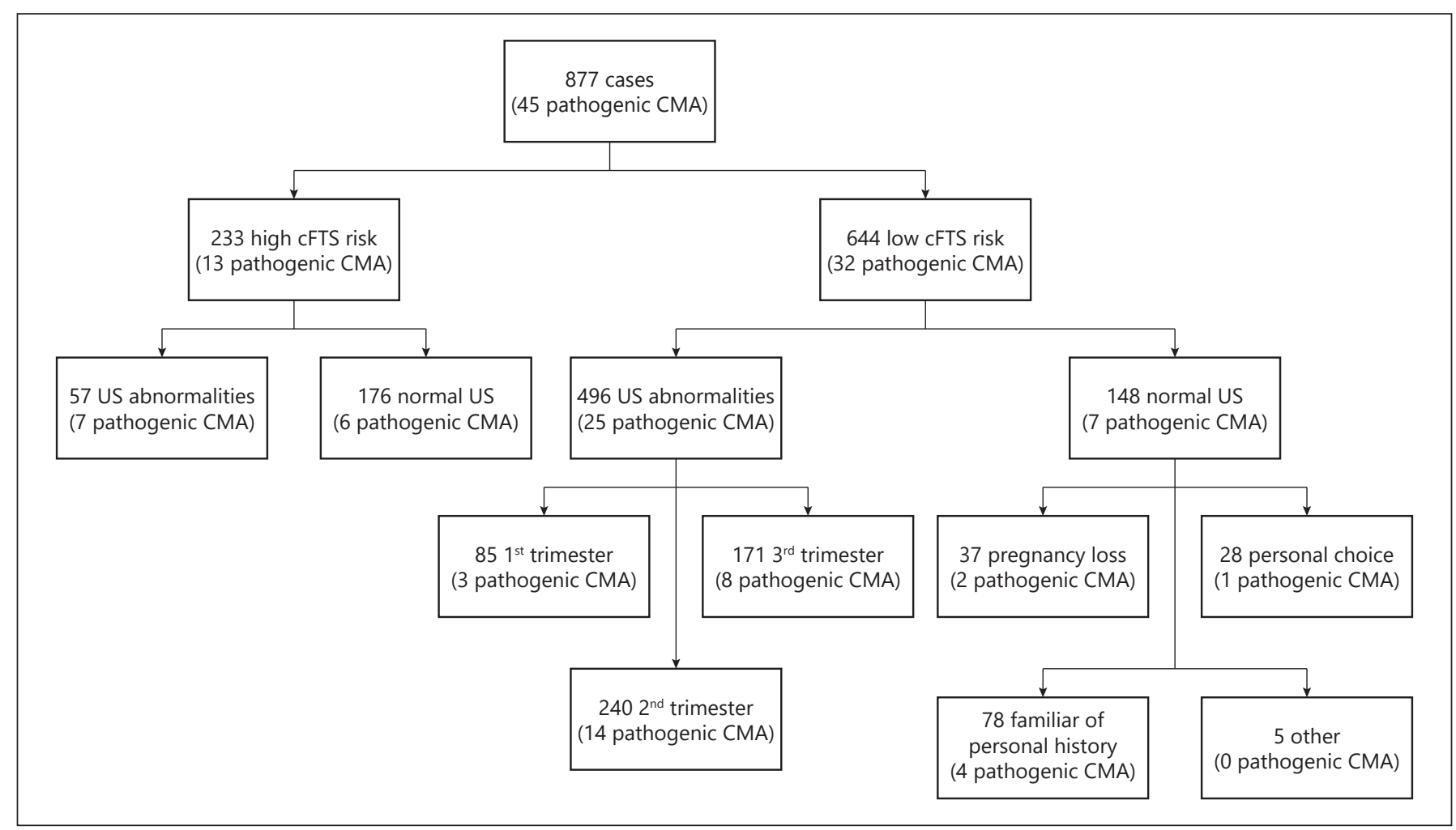

Fig. 1. Description of the study population. The number of pathogenic cases detected by CMA is shown in brackets.

Table 1.cFTS risk scores and abnormal serum biomarker frequencies in the pathogenic CMA group

\begin{tabular}{ll}
\hline Abnormal value & Pathogenic CMA $(n=45)$ \\
\hline cFTS risk score, $n(\%)$ & \\
$\quad>1 / 10$ & $5(11)$ \\
$\quad 1 / 10-1 / 250$ & $8(18)$ \\
$\quad 1 / 251-1 / 1,100$ & $8(18)$ \\
$\quad<1 / 1,100$ & $24(53)$ \\
Abnormal $\beta$-hCG, $n(\%)$ & \\
$\quad \leq 0.37$ MoM & $8(18)$ \\
$\quad \geq 2.51$ MoM & $2(4)$ \\
Abnormal PAPP-A, $n(\%)$ & \\
$\quad \leq 0.36$ MoM & $4(9)$ \\
$\quad \geq 2.46$ MoM & $1(2)$ \\
Abnormal fetal NT, $n(\%)$ & $8(18)$ \\
$\quad \geq 3.5$ mm & \\
\hline
\end{tabular}

cFTS risk $\geq 1 / 250$ and for women who had low serum $\beta$-hCG levels $(\leq 0.37 \mathrm{MoM})$ or an increased fetal NT $(\geq 3.5$ $\mathrm{mm}$ ) (Table 3). The risk of atypical chromosomal aberrations occurring in pregnancies in the high-risk cFTS group raised when increased fetal NT or low maternal serum $\beta$-hCG levels were detected; from 6 to $10 \%$ when fetal NT was considered and up to $20 \%$ for women with low serum $\beta$-hCG levels. We further examined the prevalence of atypical chromosomal aberrations not being detected by US in the first or second trimester of gestation. A similar increase in the prevalence of clinically significant CNVs was observed in the high-risk cFTS group when increased fetal NT or low serum $\beta$-hCG levels were detected. Finally, in our cohort the residual risk for an atypical aberration being missed by US in the high-risk cFTS group was $0.9 \%(2 / 233)$.

To explore the impact of different screening approaches on the detection of atypical chromosomal aberrations, theoretical detection rates were estimated according to 3 main screening strategies: (1) a conventional strategy, in which invasive testing is offered to all women with a cFTS risk $\geq 1 / 250$; (2) a contingent strategy, in which invasive testing is offered to all women with a cFTS risk $>1 / 10$ and prenatal cfDNA screening to women with a cFTS risk between 1/10-1/1,100; (3) and primary cfDNA screening strategy, in which prenatal cfDNA screening is offered to all women without performing cFTS testing. For each strategy, detection rates were calculated including in- 
Table 2. Comparison of abnormal biomarker frequencies ( $\beta-h C G$, PAPP-A, and fetal NT) between women in the normal and abnormal CMA group

\begin{tabular}{llllll}
\hline & Range & $\begin{array}{l}\text { Abnormal CMA } \\
(n=45), n(\%)\end{array}$ & $\begin{array}{l}\text { Normal CMA } \\
(n=832), n(\%)\end{array}$ & $\begin{array}{l}\text { Or } \\
(95 \% \mathrm{Cl})\end{array}$ & $p$ value \\
\hline$\beta-\mathrm{hCG}, \mathrm{MoM}$ & $\leq 0.37$ & $8(17.8)$ & $63(7.6)$ & $2.62(1.17-5.89)$ & 0.02 \\
& $\begin{array}{l}0.38-2.50 \\
\geq 2.51\end{array}$ & $\begin{array}{l}35(77.8) \\
2(4.4)\end{array}$ & $\begin{array}{l}722(86.8) \\
47(5.6)\end{array}$ & $0.88(0.20-3.76)$ & 1.00 \\
\hline PAPP-A, MoM & $\leq 0.36$ & $4(8.9)$ & $87(10.5)$ & $0.63(0.03-3.05)$ & 1.00 \\
& $0.37-2.45$ & $40(88.9)$ & $713(85.7)$ & & \\
& $\geq 2.46$ & $1(2.2)$ & $32(3.8)$ & $0.56(0.07-4.18)$ & 1.00 \\
\hline Fetal NT, mm & $<3.5$ & $\begin{array}{l}37(82.2) \\
8(17.8)\end{array}$ & $757(91.0)$ & & \\
& $\geq 3.5$ & $75(9.0)$ & $2.18(0.98-4.86)$ & 0.06 \\
\hline
\end{tabular}

Table 3. Residual risks for atypical aberrations in the high cFTS risk group, with low $\beta$-hCG or an increased fetal NT, or their combination, for all cases or for cases with normal first- or second-trimester US

\begin{tabular}{|c|c|c|c|c|c|c|c|c|c|c|}
\hline \multirow[t]{2}{*}{ Risk group } & \multirow{2}{*}{$\begin{array}{l}\text { Total } \\
\text { cases }\end{array}$} & \multicolumn{3}{|l|}{ All cases } & \multicolumn{3}{|c|}{ Normal first-trimester US } & \multicolumn{3}{|c|}{ Normal second-trimester US } \\
\hline & & $\begin{array}{l}\text { atypical } \\
\text { aberrations }\end{array}$ & $\begin{array}{l}\text { prevalence } \\
\text { atypical } \\
\text { aberrations, \% }\end{array}$ & $\begin{array}{l}\text { residual } \\
\text { risk }\end{array}$ & $\begin{array}{l}\text { atypical } \\
\text { aberrations }\end{array}$ & $\begin{array}{l}\text { prevalence } \\
\text { atypical } \\
\text { aberrations, \% }\end{array}$ & $\begin{array}{l}\text { residual } \\
\text { risk }\end{array}$ & $\begin{array}{l}\text { atypical } \\
\text { aberrations }\end{array}$ & $\begin{array}{l}\text { prevalence } \\
\text { atypical } \\
\text { aberrations, \% }\end{array}$ & $\begin{array}{l}\text { residual } \\
\text { risk }\end{array}$ \\
\hline $\mathrm{cFTS}>1 / 10$ & 53 & 5 & 9.4 & 1 in 11 & 2 & 3.7 & 1 in 27 & 1 & 1.9 & 1 in 53 \\
\hline cFTS $\geq 1 / 250$ & 233 & 13 & 5.6 & 1 in 18 & 6 & 2.6 & 1 in 39 & 2 & 0.9 & 1 in 117 \\
\hline Fetal NT $\geq 3.5 \mathrm{~mm}$ & 83 & 8 & 9.6 & 1 in 10 & 4 & 4.8 & 1 in 21 & 2 & 2.4 & 1 in 42 \\
\hline cFTS $\geq 1 / 250+$ fetal NT $\geq 3.5 \mathrm{~mm}$ & 82 & 8 & 9.8 & 1 in 10 & 4 & 3.7 & 1 in 21 & 2 & 2.4 & 1 in 41 \\
\hline$\beta$-hCG $\leq 0.37 \mathrm{MoM}$ & 71 & 8 & 11.3 & 1 in 9 & 6 & 8.5 & 1 in 12 & 1 & 1.4 & 1 in 71 \\
\hline$c F T S \geq 1 / 250+\beta-h C G \leq 0.37$ MoM & 15 & 3 & 20.0 & 1 in 5 & 1 & 8.3 & 1 in 15 & 0 & 0.0 & - \\
\hline
\end{tabular}

Table 4. Detection rates of CNV in the first and second trimester by different screening approaches

\begin{tabular}{|c|c|c|c|c|c|}
\hline Strategy & $\begin{array}{l}\text { Detection rate } \\
\text { per cFTS risk } \\
\text { score approach, } \\
n(\%)\end{array}$ & $\begin{array}{l}\text { Additional cases } \\
\text { detected when } \\
\text { considering fetal NT } \\
\geq 3.5 \mathrm{~mm}, n(\%)\end{array}$ & $\begin{array}{l}\text { Additional cases } \\
\text { detected when } \\
\text { considering } \beta \text {-hCG } \\
\leq 0.37 \text { MoM, } n(\%)\end{array}$ & $\begin{array}{l}\text { Additional cases } \\
\text { by first-trimester } \\
\text { US anomaly, } n(\%)\end{array}$ & $\begin{array}{l}\text { First- } \\
\text { trimester } \\
\text { detection } \\
\text { rate, } n(\%)\end{array}$ \\
\hline $\begin{array}{l}\text { Conventional strategy } \\
\text { IT } \geq 1 / 250\end{array}$ & $13(29)$ & $0(0)$ & $5(11)$ & $1(2)$ & $19(42)$ \\
\hline $\begin{array}{l}\text { Contingent strategy } \\
\text { IT >1/10 } \\
\text { cfDNA } 1 / 10-1 / 1,100\end{array}$ & $5(11)$ & $3(7)^{a}$ & $5(11)^{a}$ & $4(9)$ & $15(33)$ \\
\hline $\begin{array}{l}\text { Primary strategy } \\
\text { Only prenatal cfDNA screening }\end{array}$ & $0(0)$ & $8(18)$ & $0(0)^{b}$ & $4(9)$ & $12(27)$ \\
\hline
\end{tabular}

IT, invasive testing. ${ }^{a}$ In 2 cases NT was $\geq 3.5 \mathrm{~mm}$ and $\beta$-hCG was $\leq 0.37$. These cases are included both in counts, however, in the firsttrimester detection rate they have only been considered once. ${ }^{b} \beta-h C G$ was not considered in this strategy since it would not be determined.

creased fetal NT and low $\beta$-hCG levels as additional biomarkers, and first-trimester US anomaly other than fetal NT. As shown in Table 4, the percentage of detected atypical chromosomal aberrations detected in the first-trimester of gestation decreased in those approaches that include prenatal cfDNA screening. However, when considering fetal NT and low $\beta$-hCG maternal serum levels, these percentages improve significantly with both biomarkers contributing to identifying up to $20 \%$ more cases (8/45) harboring clinically significant CNV. 
Table 5. Frequencies of the general population by cFTS risk and additional biomarkers

\begin{tabular}{lll}
\hline Risk group & $\begin{array}{l}\text { Singleton pregnancies } \\
(n=3,970), n(\%)\end{array}$ & $\begin{array}{l}\text { Cases/ } \\
\text { year }\end{array}$ \\
\hline $1 / 10$ & $27(0.7)$ & 14 \\
$+\beta$-hCG $\leq 0.37$ & $10(0.3)$ & 5 \\
+ Fetal NT $\geq 3.5$ & $24(0.6)$ & 12 \\
$1 / 10-1 / 250$ & $170(4.3)$ & 85 \\
$+\beta$-hCG $\leq 0.37$ & $4(0.1)$ & 2 \\
+ Fetal NT $\geq 3.5$ & $12(0.3)$ & 6 \\
$1 / 251-1 / 1,100$ & $522(13.1)$ & 261 \\
$+\beta$-hCG $\leq 0.37$ & $6(0.2)$ & 3 \\
+ Fetal NT $\geq 3.5$ & $2(0.05)$ & 1 \\
$<1,100$ & $3,251(81.9)$ & 1,626 \\
$+\beta$-hCG $\leq 0.37$ & $183(4.6)$ & 92 \\
+ Fetal NT $\geq 3.5$ & $1(0.03)$ & 1 \\
\hline
\end{tabular}

Data obtained from all singleton pregnancies $(n=3,970)$ who were underwent cFTS at our hospital through 2018 and 2019.

Finally, we assessed the burden of additional invasive procedures if fetal NT and low maternal serum $\beta$-hCG levels were considered in the contingent screening approach (Table 5). For this proposal, data obtained from singleton pregnancies $(n=3,970)$ over a 2 -year period (2018-2019) at our institution were collected. The results showed that 105 pregnancies per year (5.3\%) fulfill these criteria.

\section{Discussion/Conclusion}

In this study, we have analyzed the association between cFTS and atypical chromosomal aberrations undetectable by prenatal cfDNA screening, in order to assess the impact of the introduction of prenatal cfDNA testing on their detection rates. Our data demonstrate that almost a $30 \%$ of clinically relevant chromosomal aberrations detected by CMA analysis at our institution were at high-risk for Down syndrome $(\geq 1 / 250)$. Furthermore, results have proved that low maternal serum $\beta$-hCG levels $(\leq 0.37 \mathrm{MoM})$ and increased fetal NT $(\geq 3.5 \mathrm{~mm})$ can contribute to better stratify pregnancies that may benefit from invasive testing over cfDNA testing.

Although cFTS was initially designed to identify pregnancies at increased risk of Down syndrome [3], it has now been shown that it can also be useful in identifying other atypical chromosomal abnormalities $[10,11,13$, 14]. In fact, the prevalence of atypical chromosomal abnormalities with a high-risk score for Down syndrome in
cFTS screening has been documented in other studies, ranging from 32 to $55 \%$ for a cutoff value varying between $1 / 200$ and $1 / 300$ [11, 13, 14]. In clinical series of pregnancies with increased risk in cFTS, the highest risk for abnormal findings are detected in pregnancies in the very high-risk cFTS group $(>1 / 10,>1 / 50)$; however, a substantial number of abnormal findings are still detected in the high-risk group (from $1 / 10$ or $1 / 50$ to $1 / 250$ ) (i.e., [20]). Our results are consistent with these observations, supporting the idea that the conventional cFTS algorithm can potentially identify one-third of the atypical chromosomal abnormalities (Table 1).

The implementation of prenatal cfDNA testing has changed the traditional approach of prenatal screening, and although it depends on the strategy adopted by each clinical center, prenatal cfDNA analysis is mainly used as an alternative to invasive testing. New research has focused on the detection of CNVs and other aneuploids from cfDNA screening data. These data showed that their detection is closely related to the size of the CNV, and at least $5 \mathrm{Mb}$ or $10 \mathrm{Mb}$ is needed to achieve an adequate sensitivity $[21,22]$. Based on this assumption, in our cohort the detection rate by NIPT would have been 12 of the 45 CNVs (26.7\%) whose size exceeded $5 \mathrm{Mb}$. Sequencing depth also plays a role, however, higher sequencing depth is also associated with higher cost, not always feasible for large population screening programs. Although expanded NIPT offers the opportunity to identify other chromosomal aberrations in addition to common aneuploidies, a portion of atypical chromosomal aberrations will remain undetected.

In an attempt to look for further factors that may assist in screening for such conditions, we assessed the role of individual cFTS biomarkers in providing information on the risk of atypical chromosomal aberrations. Our results showed that low maternal serum $\beta$-hCG levels $(\leq \mathrm{p} 5)$ and an increased fetal NT $(\geq 3.5 \mathrm{~mm})$ are associated with the presence of an atypical chromosomal abnormality. Indeed, the residual risk of a clinically significant CNV is 3 times higher when low $\beta$-hCG levels are detected or 2 times higher when fetal NT $\geq 3.5 \mathrm{~mm}$ is detected in highrisk pregnancies (cFTS $\geq 1 / 250$ ) (Table 2 ). A similar association has been previously reported by Bornstein et al. [18]. Contrary, the relationship between serum PAPP-A levels with CNVs is still unclear. Although large population-based studies have reported an association with low PAPP-A levels, neither Bornstein et al. [18] nor our study have identified a significant association. This difference could be due to an effect of the sample size of the included cohort or the defined PAPP-A cutoff point. 
On the other hand, the association between an increased fetal NT and atypical chromosome aneuploidies is well documented [14,23-25]. The prevalence of atypical chromosomal aberrations has been found to be significantly higher when fetal NT is $\geq 3.5 \mathrm{~mm}$, although some studies suggest that the cutoff point of fetal NT for invasive testing could be $3 \mathrm{~mm}$ rather than of $3.5 \mathrm{~mm} \mathrm{[26,}$ 27]. In our center, we use a customized ninety-ninth percentile ranging from $2.27 \mathrm{~mm}$ at $45 \mathrm{~mm}$ CRL to $3.78 \mathrm{~mm}$ at $84 \mathrm{~mm}$ CRL. It is noteworthy that in our cohort, all cases with an increased fetal NT had a cFTS risk $\geq 1 / 250$. Therefore, fetal NT did not provide additional information if cFTS risk $\geq 1 / 250$ was considered as a referral criterion for invasive testing but might be considered as an important criterion for prioritizing invasive testing over prenatal cfDNA testing. In fact, according to the current updated International Society of Ultrasound in Obstetrics and Gynecology (ISUOG) consensus statement, cfDNA testing should not replace first-trimester US and should not be offered when an US anomaly or markedly increased NT is detected [28].

Counseling for invasive testing for fetal chromosome aberrations has changed and varies significantly between counties [8]. While some countries have adopted prenatal cfDNA screening as a first-line test (primary screening), others have implemented it as an adjunct to the existing screening programs (secondary and contingent strategies). Based on the data presented, we assessed the residual risks of atypical chromosomal abnormalities in high-risk pregnancies considering low serum $\beta$-hCG levels and increased fetal NT. In our setting, this is of great interests, as a contingent cfDNA prenatal screening approach has been adopted, where high-risk pregnancies $(1 / 10-1 / 250)$ can choose between a cfDNA test and a CMA after an invasive procedure. In our cohort, the residual risk for high-risk pregnancies increased from 1:18 to $1: 5$ for women with low $\beta$-hCG levels and from $1: 18$ to 1:10 for those with increased fetal NT (Table 3). Given that the majority of the detected fetal structural abnormalities were identified with the second-trimester US, our results point out that altered serum $\beta$-hCG levels can have a substantial impact on early detection of clinically relevant CNVs.

Finally, we examined the detection rates of pathogenic CNVs in the first trimester of pregnancy in different clinical scenarios according to the different screening approaches (Table 4). In light of our results, the detection rate of atypical chromosomal abnormalities if the contingent strategy is followed is 2.5 times lower than the traditional approach. However, when considering the mater- nal serum $\beta$-hCG levels, the detection rate increased and reached similar percentage as in the conventional approach. On the other hand, and as expected, using prenatal cfDNA screening as the first-line of prenatal screening will have missed all atypical chromosomal abnormalities included in our cohort. However, when considering fetal NT, the detection rates of pathogenic CNVs in the first trimester will increase up to $18 \%$ (Table 4). This result reinforces the consensus that prenatal cfDNA screening should always be offered in combination with US $[29,30]$.

Our study had some limitations. Although the study group was considerably large, the number of cases with alterations by CMA testing was small. Moreover, our study only included women who were referred or who decided to undergo invasive testing, and therefore there may be a selection bias.

In conclusion, in this study, we have demonstrated that a substantial proportion of atypical chromosome aberrations are potentially detectable by cFTS screening, and that low serum $\beta$-hCG levels and an increased fetal NT can be considered independent risk factors for CNVs. Our findings may help clinicians and women to decide whether invasive testing or prenatal cfDNA screening tests are more appropriate for each situation.

\section{Statement of Ethics}

The study was approved by the Institutional Ethical Committee on January 24, 2020 (HCB/2019/1029). All procedures followed were in accordance with the ethical standards of the responsible committee on human experimentation and with the Helsinki Declaration. Written informed consent was not required due to the observational and retrospective nature of the study, as approved by the Institutional Ethics Committee of the Hospital Clínic de Barcelona.

\section{Conflict of Interest Statement}

The authors have no conflicts of interest to declare.

\section{Funding Sources}

This work was supported by Fundación Alicia Koplowitz (AKOPLOWITZ18_001) and AGAUR from the Autonomous Catalan Government (2017SGR1134). The CIBER de Enfermedades Raras is an initiative of the Instituto de Salud Carlos III. 


\section{Author Contributions}

R.W. and L.R.R. designed the concept of the study. R.W., L.R.R., and E.C. performed the data collection, and R.W. performed the data analysis. R.W. and L.R.R. wrote the first draft. E.C., I.Me., J.L., I.Ma., C.B., and A.B. reviewed the manuscript. All the authors read and approved the final manuscript and confirmed authorship.

\section{References}

1 Wapner R, Thom E, Simpson JL, Pergament E, Silver R, Filkins K, et al. First-trimester screening for trisomies 21 and 18. N Engl J Med. 2003 Oct 9;349(15):1405-13.

2 Nicolaides KH. First-trimester screening for chromosomal abnormalities. Semin Perinatol. 2005;29(4):190-4.

3 Spencer K, Souter V, Tul N, Snijders R, Nicolaides KH. A screening program for trisomy 21 at 10-14 weeks using fetal nuchal translucency, maternal serum free beta-human chorionic gonadotropin and pregnancy-associated plasma protein-A. Ultrasound Obstet Gynecol. 1999;13(4):231-7.

4 Spencer K, Nicolaides KH. A first trimester trisomy 13/trisomy 18 risk algorithm combining fetal nuchal translucency thickness, maternal serum free beta-hCG and PAPP-A. Prenat Diagn. 2002;22(10):877-9.

5 Kagan KO, Wright D, Maiz N, Pandeva I, Nicolaides KH. Screening for trisomy 18 by maternal age, fetal nuchal translucency, free beta-human chorionic gonadotropin and pregnancy-associated plasma protein-A. Ultrasound Obstet Gynecol. 2008;32(4):488-92.

6 Norton ME, Jacobsson B, Swamy GK, Laurent LC, Ranzini AC, Brar H, et al. Cell-free DNA analysis for noninvasive examination of trisomy. New Engl J Med. 2015;372(17):1589-97.

7 Gil MM, Accurti V, Santacruz B, Plana MN, Nicolaides KH. Analysis of cell-free DNA in maternal blood in screening for aneuploidies: updated meta-analysis. Ultrasound Obstet Gynecol. 2017;50(3):302-14.

8 Gadsbøll K, Petersen OB, Gatinois V, Strange $\mathrm{H}$, Jacobsson B, Wapner R, et al. Current use of noninvasive prenatal testing in Europe, Australia and the USA: A graphical presentation. Acta Obstet Gynecol Scand. 2020;99(6) $722-30$.

9 Wellesley D, Dolk H, Boyd PA, Greenlees R, Haeusler M, Nelen V, et al. Rare chromosome abnormalities, prevalence and Prenat Diagn rates from population-based congenital anomaly registers in Europe. Eur J Hum Genet. 2012;20(5):521-6.

10 Alamillo CML, Krantz D, Evans M, Fiddler M, Pergament E. Nearly a third of abnormalities found after first-trimester screening are different than expected:10-year experience from a single center. Prenat Diagn. 2013;33(3):251-6.

11 Lindquist A, Poulton A, Halliday J, Hui L. Prenatal diagnostic testing and atypical chromosome abnormalities following combined first-

\section{Data Availability Statement}

The data that support the findings of this study are not publicly available due to their containing information that could compromise the privacy of research participants but are available from the corresponding author (LRR) upon reasonable request. trimester screening: implications for contingent models of non-invasive prenatal testing. Ultrasound Obstet Gynecol. 2018;51(4):48792.

12 Norton ME, Jelliffe-Pawlowski LL, Currier RJ. Chromosome abnormalities detected by current prenatal screening and noninvasive prenatal testing. Obstet Gynecol. 2014;124(5): 979-86.

13 Iwarsson E, Conner P. Detection rates and residual risk for a postnatal diagnosis of an atypical chromosome aberration following combined first-trimester screening. Prenat Diagn. 2020;40(7):852-9.

14 Petersen OB, Vogel I, Ekelund C, Hyett J, Tabor A. Potential diagnostic consequences of applying non-invasive prenatal testing: population-based study from a country with existing first-trimester screening. Ultrasound $\mathrm{Ob}$ stet Gynecol. 2014;43(3):265-71.

15 Wulff CB, Gerds TA, Rode L, Ekelund CK, Petersen OB, Tabor A. Risk of fetal loss associated with invasive testing following combined first-trimester screening for down syndrome: a national cohort of 147,987 singleton pregnancies. Ultrasound Obstet Gynecol. 2016; 47(5):38-44.

16 Hui L, Hutchinson B, Poulton A, Halliday J. Population-based impact of noninvasive prenatal screening on screening and diagnostic testing for fetal aneuploidy. Genet Med. 2017; 19(12):1338-45.

17 Larion S, Warsof SL, Romary L, Mlynarczyk M, Peleg D, Abuhamad AZ. Uptake of noninvasive prenatal testing at a large academic referral center. Am J Obstet Gynecol. 2014; 211(6):651e1-7.

18 Bornstein E, Gulersen M, Krantz D, Cheung SW, Maliszewski K, Divon MY. Microarray analysis: First-trimester maternal serum free $\beta$-hCG and the risk of significant copy number variants. Prenat Diagn. 2018;38(12):971-8.

19 Richards S, Aziz N, Bale S, Bick D, Das S, Gastier-Foster J, et al. Standards and guidelines for the interpretation of sequence variants: a joint consensus recommendation of the American College of Medical Genetics and Genomics and the Association for Molecular Pathology. Genet Med. 2015;17(5):405-24.

20 Vogel I, Petersen OB, Christensen R, Hyett J, Lou S, Vestergaard EM. Chromosomal microarray as primary diagnostic genomic tool for pregnancies at increased risk within a population-based combined first-trimester screening program. Ultrasound Obstet Gynecol. 2018 Apr 1;51(4):480-6.

21 Chen Y, Lai Y, Xu F, Qin H, Tang Y, Huang $\mathrm{X}$, et al. The application of expanded noninvasive prenatal screening for genome-wide chromosomal abnormalities and genetic counseling. J Matern Fetal Neonatal Med. 2021;34:2710-6

22 Wang J, Zhang B, Zhou L, Zhou Q, Chen Y, Yu B. Comprehensive evaluation of non-invasive prenatal screening to detect fetal copy number variations. Front Genet. 2021;12:665589.

23 Zhao XR, Gao L, Wu Y, Wang YL. Application of chromosomal microarray in fetuses with increased nuchal translucency. J Matern Fetal Neonatal Med. 2020;33(10):1749-54.

24 Lund IC, Christensen R, Petersen OB, Vogel I, Vestergaard EM. Chromosomal microarray in fetuses with increased nuchal translucency. Ultrasound Obstet Gynecol. 2015;45(1):95100.

25 Grande M, Jansen FAR, Blumenfeld YJ, Fisher A, Odibo AO, Haak MC, et al. Genomic microarray in fetuses with increased nuchal translucency and normal karyotype: a systematic review and meta-analysis. Ultrasound $\mathrm{Ob}$ stet Gynecol. 2015;46(6):650-8.

26 Petersen OB, Smith E, van Opstal D, Polak M, Knapen MFCM, Diderich KEM, et al. Nuchal translucency of 3.0-3.4 mm an indication for NIPT or microarray? Cohort analysis and literature review. Acta Obstet Gynecol Scand. 2020;99(6):765-74.

27 Hui L, Palma-Dias R, da Silva Costa F. The diagnosis of an increased NT remains a persistent clinical dilemma that deserves a rational approach. Am J Obstet Gynecol. 2021 Oct; 225(4):464-5.

28 Salomon LJ, Alfirevic Z, Audibert F, Kagan KO, Paladini D, Yeo G, et al. ISUOG updated consensus statement on the impact of cfDNA aneuploidy testing on screening policies and prenatal ultrasound practice. Ultrasound $\mathrm{Ob}$ stet Gynecol. 2017 Jun;49(6):815-6.

29 Bardi F, Bosschieter P, Verheij J, Go A, Haak $\mathrm{M}$, Bekker M, et al. Is there still a role for nuchal translucency measurement in the changing paradigm of first trimester screening? Prenat Diagn. 2020 Jan 1;40(2):197-205.

30 Salomon LJ, Porcher R, Socolov D, Lamrani H, Ville Y. Repeat measurements of nuchal translucency at 11-14 weeks of gestation: when do we need them? Ultrasound Obstet Gynecol. 2013 Dec;42(6):629-33. 